IdeAs

Idées d'Amériques

$6 \mid 2015$

Migrer dans les Amériques

\title{
Les provinces de l'Ouest : toujours conservatrices mais avec un retour des libéraux
}

Frédéric Boily

\section{OpenEdition}

1 Journals

Édition électronique

URL : https://journals.openedition.org/ideas/1255

DOI : 10.4000 /ideas. 1255

ISSN : 1950-5701

Éditeur

Institut des Amériques

Référence électronique

Frédéric Boily, "Les provinces de l'Ouest : toujours conservatrices mais avec un retour des libéraux », IdeAs [En ligne], 6 | 2015, mis en ligne le 17 décembre 2015, consulté le 20 octobre 2022. URL : http:// journals.openedition.org/ideas/1255; DOI : https://doi.org/10.4000/ideas.1255

Ce document a été généré automatiquement le 20 octobre 2022

\section{(c) (i) (9)}

Creative Commons - Attribution - Pas d'Utilisation Commerciale - Pas de Modification 4.0 International - CC BY-NC-ND 4.0

https://creativecommons.org/licenses/by-nc-nd/4.0/ 


\title{
Les provinces de l'Ouest : toujours conservatrices mais avec un retour des libéraux
}

\author{
Frédéric Boily
}

\section{Introduction}

1 À l'automne 2014, le président français François Hollande s'est rendu au Canada pour une première visite officielle depuis François Mitterrand en $1987^{1}$. Au lieu de commencer son voyage au Québec ou dans la capitale fédérale à Ottawa, le président Hollande s'est d'abord rendu en Alberta, plus précisément au parc national de Banff qu'il a visité en compagnie du premier ministre du moment, Stephen Harper, et du gouverneur général David Johnston (The Canadian Press 2015). Cependant, le président français n'était pas là simplement pour admirer les paysages et la faune sauvage du parc national car cette visite doit être placée dans l'évolution du contexte politique et économique survenue depuis près de 20 ans (Castro-Rea et Boily, 2014). Les provinces de l'Ouest se sont en effet imposées comme étant les plus dynamiques économiquement dans la première décennie des années 2000. Aussi controversée soit-elle au plan environnemental, l'exploitation des sables bitumineux albertains a généré une importante activité économique qui a tiré la croissance canadienne vers le haut avec un marché de l'emploi très dynamique et des taux de chômage en-deçà de la moyenne canadienne, même après une année de baisse des prix du pétrole (Institut de la statistique). En débutant son voyage dans cette partie du Canada, le président Hollande semblait donc accréditer l'idée selon laquelle les provinces à l'ouest de l'Ontario étaient incontournables au plan politique, une importance que les élections générales survenues trois ans plus tôt semblaient aussi avoir confirmée. 


\section{Situation politique en 2011}

2 C'est en effet une région qui, en mai 2011, s'était bien installée au pouvoir puisqu'elle avait largement appuyé le Parti conservateur du Canada (PCC) de Stephen Harper, ce dernier étant lui-même représentant d'une circonscription albertaine de la ville de Calgary. Dans toutes les provinces de l'ouest du pays, les conservateurs avaient remporté des majorités convaincantes (voir tableau). Cela commençait d'abord par l'Alberta où les conservateurs avaient remporté 27 circonscriptions sur 28 (67\% des suffrages) et la Saskatchewan où le PCC avait remporté 13 circonscriptions sur 14 (56\% des suffrages). Au Manitoba aussi les conservateurs avaient obtenu plus de la majorité des voix (53\%), ce qui leur avait valu de se retrouver avec 11 députés sur une possibilité de 14. En Colombie-Britannique, les résultats électoraux du parti de Stephen Harper étaient moins dominants mais ils avaient tout de même gagné une majorité de sièges, soit 21 sur les 36 circonscriptions que comptait la province à ce moment grâce à un soutien de $45 \%$ du vote populaire (Boily 2011).

3 Dans ce contexte, les deux principaux partis d'opposition, à savoir le Parti libéral du Canada (PLC) qui est d'orientation centriste et le Nouveau Parti démocratique (NPD) qui est à gauche, auxquels s'ajoute le Parti vert (PV) se retrouvaient dans une situation politique difficile dans ces quatre provinces. Seuls les néo-démocrates dirigés par le chef Jack Layton, décédé d'un cancer dans l'été suivant l'élection, étaient parvenus à faire élire une quinzaine de députés, surtout en Colombie-Britannique. Quant au Parti libéral du Canada, qui était alors dirigé par l'intellectuel Michal Ignatieff, la situation était sombre pour cette formation, dominante au Canada jusqu'à l'élection du gouvernement minoritaire libéral de Paul Martin de 2004. En Alberta et en Saskatchewan, le PLC recueillait seulement $9 \%$ des voix dans chacune des deux provinces, alors que la situation était à peine meilleure en Colombie-Britannique (13\%). $\mathrm{Au}$ Manitoba, une province où les libéraux avaient connu du succès dans le passé, notamment auprès de la communauté francophone de Winnipeg, ils recueillaient seulement $17 \%$ des voix et un seul député. Et si le PV était parvenu à faire élire sa chef (Elizabeth May) dans une circonscription de l'Ile de Vancouver, en revanche, cette formation politique demeurait une force politique marginale, compte tenu d'un soutien populaire sous les $10 \%$. Bref, aux lendemains de l'élection de 2011, les deux grands partis d'opposition devaient reconstruire leur base électorale dans les provinces mentionnées plus haut.

4 Or, contre toute attente, la situation politique aura évolué dans une direction inattendue, si bien qu'au début de l'élection déclenchée précipitamment par le premier ministre le 2 août 2015, une embellie électorale paraissait possible pour eux. D'une part, la chute des prix du pétrole frappait de plein fouet les économies de l'Ouest, plus particulièrement celle de l'Alberta, faisant en sorte que le Canada se retrouvait en récession pour les six premiers mois de l'année 2015. D'autre part, l'élection provinciale albertaine, tenue le 5 mai 2015, a vu un véritable tremblement de terre politique survenir dans cette province où le Parti conservateur provincial gouvernait sans interruption depuis 1971. Les conservateurs provinciaux ont été remplacés par les néodémocrates dirigés par Rachel Notley, son parti défendant un programme interventionniste de gauche. Or, le renversement de cette dynastie provinciale autorisait les partis d'opposition à croire que leur chance de faire élire des candidats dans la région était meilleure que jamais dans un passé récent. 


\section{Le PCC reste la formation dominante dans l'Ouest}

5 Au début de l'élection, il était possible de croire à une percée importante des néodémocrates de Thomas Mulcair. Au contraire, c'est le PLC qui s'est imposé avec un gouvernement majoritaire, même dans l'Ouest où les libéraux ont mieux fait que leurs adversaires progressistes. En effet, comme le montre le tableau, le PLC est parvenu à effectuer des gains importants en Colombie-Britannique en passant de 2 sièges à 17 grâce à un bond de plus de $20 \%$ dans les intentions de vote. Il faut cependant préciser que les victoires libérales se sont produites, en Colombie-Britannique, surtout dans la région de Vancouver. Au Manitoba, c'est sensiblement la même dynamique qui s'est produite puisque les libéraux sont aussi parvenus à décrocher quelques victoires surtout dans la ville de Winnipeg. En fait, presque partout au Canada, les libéraux dominent auprès de l'électorat urbain.

D'ailleurs les quatre victoires libérales en Alberta sont survenues à Edmonton (2), et plus surprenant encore, à Calgary (2), métropole de l'industrie énergétique. En effet, dans cette dernière ville, les libéraux fédéraux n'étaient pas parvenus à obtenir une victoire depuis 1968, lorsque le PLC, alors dirigé par le père de l'actuel premier ministre, y avait fait élire un candidat. Mais par la suite, les libéraux avaient été rayés de la carte de Calgary et l'animosité, le mot n'est pas trop fort, envers le PLC avait atteint des sommets lorsque Pierre Elliot Trudeau avait lancé le Programme national d'énergie (PNE), en 1980. Ce programme avait été perçu comme ce qui avait freiné pour de bon la croissance du secteur énergétique. Pendant des décennies, la classe politique albertaine ne cessait de rappeler les fantômes de ce controversé programme pour discréditer les efforts des libéraux fédéraux et provinciaux pour faire élire des candidats en Alberta.

Tableau : Élections fédérales de 2011 et $2015^{2}$

\begin{tabular}{|l|l|l|l|l|l|l|l|}
\hline & $\begin{array}{l}\text { Résultats } \\
\text { électoraux }\end{array}$ & \multicolumn{2}{|l|}{$\begin{array}{l}\text { Prairies (Saskatchewan et } \\
\text { Manitoba) }\end{array}$} & \multicolumn{3}{l|}{ Alberta } & \multicolumn{2}{l}{$\begin{array}{l}\text { Colombie- } \\
\text { Britannique }\end{array}$} \\
\hline $\begin{array}{l}\text { Partis } \\
\text { politiques }\end{array}$ & 2011 & 2015 & 2011 & 2015 & 2011 & 2015 \\
\hline PCC & $\begin{array}{l}\text { Votes (\%) } \\
\text { Sièges (\#) }\end{array}$ & 55 & 43 & 67 & 60 & 45 & 30 \\
\hline PLC & Votes (\%) & 13 & 34 & 27 & 29 & 21 & 10 \\
\hline NPD & Sièges (\#) & 2 & 8 & 9 & 25 & 13 & 35 \\
& Votes (\%) & 29 & 19 & 0 & 4 & 2 & 17 \\
\hline PV & Sièges (\#) & 2 & 5 & 17 & 12 & 32 & 26 \\
& Votes (\%) & 3 & 1 & 1 & 12 & 14 \\
\hline
\end{tabular}

Source : Élections Canada, http://www.elections.ca/accueil.aspx 
7 Dans ce contexte, que le PLC dirigé par le fils de Pierre Trudeau soit parvenu à faire élire deux députés à Calgary a été interprété, à juste titre, comme une réussite remarquable qui venait couronner les efforts déployés par le chef libéral. Ce dernier avait soigneusement préparé le terrain avant le début de l'élection en visitant fréquemment cette province (Boily 2014). En 2012, il s'était même permis d'affirmer que le Programme national d'énergie de son père était une mauvaise politique qui avait divisé le pays, une admission audacieuse qui n'est pas étrangère à ses succès. Le succès du PLC doit beaucoup à son programme qui misait sur la promesse d'investir dans les infrastructures, ce qui plaisait à de nombreux électeurs des grandes villes qui attendaient une intervention de l'État en cette matière. Les libéraux promettaient également de rompre avec le dogme de l'équilibre budgétaire en annonçant de «modestes » déficits pour les trois prochaines années suivant leur élection.

8 Par ailleurs, l'ensemble de la région est tout de même resté fidèle aux conservateurs. Le vote «anti-Harper » qui a été très fort ailleurs au pays a été moins prononcé dans la région où le message de stabilité économique et d'équilibre budgétaire prôné par les conservateurs touchait une corde sensible auprès de l'électorat. Si le pourcentage de soutien populaire a baissé partout, même en Alberta, en revanche, en ce qui a trait au nombre de sièges, le PCC a remporté 54 sièges sur les 104 que comptent les quatre provinces. Dans les régions rurales et périurbaines, les conservateurs restent bien implantés électoralement, comme c'est le cas en Saskatchewan et dans une moindre mesure au Manitoba, alors qu'en Colombie-Britannique les 10 sièges sont cantonnés à l'intérieur de la province. Plusieurs partisans conservateurs ont ainsi l'impression d'être revenu à une situation politique similaire à celle qui prévalait après l'élection générale de 2004 lorsque le parti se retrouvait la première opposition officielle avec un parti dont la base reste dans l'Ouest.

Comme ailleurs au pays, le NPD n'a pu s'imposer comme solution de rechange aux conservateurs. Comme nous l'avons mentionné plus haut, le NPD pensait être le bénéficiaire de la vague néo-démocrate provinciale en Alberta. Mais, en misant sur un programme centriste qui promettait l'équilibre budgétaire, comme les conservateurs, le NPD laissait le champ libre aux libéraux. C'est ainsi que l'effet de relance ne s'est pas produit, les néo-démocrates devant se contenter de gains modestes de 5 circonscriptions par rapport à 2011, avec une baisse du soutien populaire dans les quatre provinces. Rien donc pour susciter de l'espoir, ce qui est aussi le cas pour les environnementalistes. Si Elizabeth May a pu se faire réélire en Colombie-Britannique, sa formation, le $\mathrm{PV}$, fait du surplace électoral en ne parvenant pas à progresser ni en regard des suffrages exprimés, ni avec l'ajout de députés supplémentaires à la Chambre des communes. Pour ces deux formations politiques, les lendemains de l'élection sont particulièrement amers au contraire des conservateurs qui peuvent toujours espérer revenir au pouvoir pour autant qu'ils parviennent de nouveau à élargir leur base électorale en-dehors de l'Ouest canadien.

\section{Conclusion}

10 À l'élection générale de 2011, plusieurs observateurs estimaient que la région de l'Ouest et tout particulièrement l'Alberta, était "in », c'est-à-dire que les conservateurs de l'endroit étaient finalement parvenus à s'installer au gouvernement à Ottawa. Des observateurs avaient décrit cette victoire comme étant un Big Shift (Bricker et Ibbitson 
2013). Rappelons que les tenants de la thèse du grand changement parlaient d'un Canada devenu plus conservateur et tourné vers le Pacifique, en raison du poids économique et démographique de l'Ouest canadien. S'ils ne croyaient pas que les conservateurs demeureraient au pouvoir de manière permanente, en revanche, affirmaient-ils, le parti qui reviendrait au pouvoir ne pourrait le faire sans adopter les principes politiques des conservateurs. Or, les résultats de l'élection du 19 octobre 2015 pourraient laisser entendre que le changement de polarité politique annoncé en direction de l'Ouest était quelque peu prématuré. Force est de constater que le scénario envisagé ne se déroule pas tout à fait comme prévu puisque les libéraux ont adopté un discours économique différent des conservateurs, tout en gagnant avec l'appui des provinces centrales. Toutefois, les auteurs mentionnés plus haut défendent toujours leur thèse. Aux lendemains des élections, ils ont soutenu que le changement vers un Canada plus conservateur est toujours en cours et que le chef libéral Justin Trudeau devra rester attentif à cette évolution de fond, notamment parce que la croissance de la population et de l'immigration qui l'accompagne ne cesse de faire croitre l'influence des provinces de l'Ouest (Bricker et Ibbitson 2015). En somme, il serait trop tôt pour dire que le Canada est retourné à son identité libérale d'antan ou encore que l'Ouest se retrouve, comme hier, marginalisé au sein de l'ensemble canadien.

\section{BIBLIOGRAPHIE}

Bricker, Darrell et John Ibbitson, The Big Shift. The seismic change in Canadian Politics, Business, and Culture and what it means for our future, Toronto, Harper Collins Publishers Ltd, 2013.

Bricker, Darrell et John Ibbitson, “Why Canada's shift to conservatism isn't dead”, The Globe and Mail, 28 octobre 2015, p. A12.

Boily, Frédéric, «L’Ouest est 'in' finalement », Policy options / Options politiques, juin 2011.

Boily, Frédéric, De Pierre à Justin Trudeau. Portrait de famille de l'idéologie libérale du Canada, 1968-2013, Québec, Presses de l'Université Laval, 2014.

Castro-Rea, Julián et Frédéric Boily (dir.), Le fédéralisme selon Harper. La place du Québec dans le Canada conservateur, Québec, Presses de l'Université Laval, 2014.

Institut de la statistique, « Comparaisons interprovinciales », http://www.stat.gouv.qc.ca/docs$\mathrm{hmi} /$ statistiques/economie/comparaisons-economiques/interprovinciales/chap6.pdf, page consultée le 27 octobre 2015.

The Canadian Press, "Francois Hollande, French president, makes 1st official visit to Western Canada", CBC News, http://www.cbc.ca/news/canada/calgary/francois-hollande-frenchpresident-makes-1st-official-visit-to-western-canada-1.2821267, page consultée le 2 août 2015. 


\section{NOTES}

1. Le président Nicholas Sarkozy avait cependant assisté au sommet Canada-UE, en 2008, à Québec.

2. La carte électorale est passée de 308 circonscriptions, en 2011, à 338, en 2015, soit 15 de plus en Ontario, 6 respectivement en Alberta et en Colombie-Britannique et 3 au Québec.

\section{AUTEUR}

\section{FRÉDÉRIC BOILY}

Frédéric Boily est professeur de science politique à la Faculté Saint-Jean de l'Université de l'Alberta (Canada) depuis 2004. Il a aussi été directeur de l'Institut d'études canadiennes (Faculté Saint-Jean) de 2013 à 2015. Auteur de plusieurs ouvrages sur la politique canadienne, il s'intéresse tout particulièrement à l'évolution de la droite canadienne et québécoise. fboily@ualberta.ca 